\title{
Detección de factores de riesgo cardiovascular en adultos de Chaco y Corrientes
}

Bioq. Cesario, A. Macarena; Esp. Goicoechea, Patricia; Bioq. Leyes Pedrozo, Ariel; Bioq. Serrano, Natalia $\left(^{*}\right)$

Resumen: Las enfermedades cardiovasculares $(\mathrm{ECV})$ son la principal causa de muerte en todo el mundo. Cada año mueren más personas por ECV que por cualquier otra causa. Son enfermedades de etiología multifactorial y su evolución está ligada a la presencia de factores de riesgo cardiovascu$\operatorname{lar}(\mathrm{FRCV})$.

Para este estudio se realizó un estudio de corte transversal, durante los años 2016 y 2017, en una muestra de 59 individuos (48 mujeres y 11 hombres). La muestra incluye a pobladores de zonas rurales aledañas a las localidades de Corrientes y Chaco donde se instalaron puestos bioquímicos como servicio a la comunidad.

Previo consentimiento informado, se tomaron muestras de sangre venosa, para determinar glucemia y el perfil lipídico. En forma paralela se realizó una encuesta, se tomaron medidas antropométricas y de presión arterial.
De acuerdo a la encuesta realizada se logró recabar que el sedentarismo y la obesidad comparten el primer lugar de prevalencia en la población estudiada. E1 73\% reportó realizar actividad física menos de 30 minutos, tres veces por semana, lo cual se consideró como indicador de sedentarismo. E1 46\% de la población analizada presentó un IMC mayor al 29,9\% indicando obesidad.

La alta prevalencia de factores de riesgo cardiovascular en adultos jóvenes remarca la importancia de realizar campañas de concientización y prevención encaminadas a reducirlos. Un alto porcentaje de la población incluida en el estudio presentó obesidad y sedentarismo esto señala la necesidad de educar a la población para mejorar los hábitos alimenticios y aumentar la actividad física.

Palabras clave: enfermedades cardiovasculares, factores de riesgo, estilo de vida.

(*) Laboratorio de Bioquímica Aplicada, Facultad de Ciencias Exactas, Naturales y Agrimensura, UNNE. Corrientes, 


\section{Objetivos}

- Valorar el estado nutricional general de la población elegida a través de parámetros antropométricos.

- Detectar factores de riesgo asociados a enfermedades cardiovasculares a través de encuestas y parámetros bioquímicos.

- Comprobar si existe asociación factores de riesgo y enfermedades cardiovasculares en la población estudiada.

\section{Introducción}

Las enfermedades cardiovasculares (ECV) son la principal causa de muerte en todo el mundo. Cada año mueren más personas por $\mathrm{ECV}$ que por cualquier otra causa. Son enfermedades de etiología multifactorial y su evolución está ligada a la presencia de factores de riesgo cardiovascular (FRCV). Desde el punto de vista epidemiológico un factor de riesgo es una condición o característica de un individuo o población que está presente en forma temprana en la vida y se asocia con un riesgo aumentado de desarrollar una enfermedad futura. En su desarrollo, intervienen diversos factores, no sólo genéticos sino también los relacionados con hábitos y estilos de vida no saludables. En el caso de los factores de riesgo cardiovascular, algunos de ellos, como la edad, el género y la historia familiar no se pueden cambiar, es decir, no son modificables. Otros, como el colesterol elevado, la hipertensión arterial, la diabetes, el tabaquismo, la obesidad, el sedentarismo y el estrés, son factores de riesgo modificables, que sí pueden ser prevenidos, tratados o modificados. Es importante destacar que mientras más factores de riesgo tenga una persona, mayor es la probabilidad de desarrollar enfermedades cardiovasculares. Por eso es fundamental que la educación para la salud apunte a evitar la adquisición de aquellas conductas que favorezcan el desarrollo de estos factores de riesgo.

La hipertensión arterial (HTA) se define como la elevación crónica de la presión arterial sistólica y/o diastólica, por encima de los valores considerados normales. (Guía de diagnóstico y manejo de la Org. Panamericana de la salud). La hipertensión arterial es considerada una de las enfermedades de la civilización y su origen es multifactorial. Es una enfermedad poligénica interrelacionada con varios factores ambientales, que ha ido en aumento con los nuevos estilos de vida inadecuados, la vida sedentaria y los hábitos alimentarios que tienden a comidas rápidas y alimentos clasificados como chatarra, de poco valor nutricional, con exceso de sal y grasas saturadas.

La diabetes es una enfermedad caracterizada por un alto nivel de glucosa resultado de defectos en la capacidad del cuerpo para producir o usar insulina. Es una de las enfermedades con mayor impacto sociosanitario, no sólo por su elevada frecuencia, sino, sobre todo, por las consecuencias de las complicaciones crónicas que comporta esta enfermedad, el importante papel que desempeña como factor de riesgo de aterosclerosis y de patología cardiovascular.

E1 consumo de tabaco constituye un problema de salud pública de carácter prioritario, pues comporta niveles altos de morbilidad y mortalidad prevenibles. A pesar 
de la existencia de campañas y programas de prevención, entre un tercio y la mitad de los escolares de países desarrollados experimentan con el tabaco antes de finalizar la educación obligatoria. Los efectos en la salud cardiovascular aparecen enseguida al empezar a fumar y son también los primeros que se revierten al dejar de fumar.

La dislipidemia es un término que empleamos para definir el aumento de la concentración plasmática de colesterol y lípidos en la sangre, es una condición que se encuentra asociada al desarrollo de una gran cantidad de padecimientos crónico degenerativo como obesidad, hipertensión, diabetes mellitus, enfermedades cardiovasculares y otros.

Es un término genérico para denominar cualquier situación clínica en la cual existan concentraciones anormales de colesterol: colesterol total (Col-total), colesterol de alta densidad (Col-HDL), colesterol de baja densidad (Col-LDL) o triglicéridos (TG). Constituyen un factor de riesgo mayor y modificable de enfermedades cardiovasculares (CV). (MINSAL).

El riesgo de padecer ateroesclerosis aumenta en consonancia con la concentración de colesterol total, incluso si esta no es lo suficientemente alta como para considerarlo dislipidemia.

La obesidad es una enfermedad producto de la interacción de factores genéticos y medioambientales. Se considera que las principales causas son los cambios en la alimentación y un estilo de vida sedentario. A partir de la segunda mitad del siglo
XX hemos modificado tanto nuestra dieta como nuestro estilo de vida. De esta forma, la disminución del consumo de frutas y verduras, ricos en fibras, y el aumento de grasas y azúcares refinados propio de las comidas rápidas, acompañados por la falta de ejercicio, impactan gravemente sobre la salud de nuestro corazón. Según la Organización Mundial de la Salud (OMS), el sobrepeso y la obesidad son condiciones en las que se presenta acumulación anormal o excesiva de grasa corporal que puede ser perjudicial para la salud. La obesidad se considera un factor de riesgo importante para la enfermedad cardiovascular y está asociada con el desarrollo de hiperinsulinemia, resistencia a la insulina, intolerancia a la glucosa, hipertensión arterial y síndrome metabólico.

La obesidad se mide por el Índice de masa corporal (IMC), que relaciona el peso y la talla, basada en la fórmula:

$$
I M C=\text { Peso }(K g) / \text { Altura }^{2}(\mathrm{~m})
$$

La Organización Mundial de la Salud considera al sedentarismo como una de las principales causas de enfermedad cardiovascular y afirma que más del 60\% de la población mundial es sedentaria, definiendo como tal a aquella persona que no realiza al menos, 30 minutos diarios o suma $150 \mathrm{mi}-$ nutos semanales de actividad física regular. Está comprobado que realizar más de 150 minutos (2 horas y 30 minutos) de actividad física moderada o una hora de actividad física vigorosa semanalmente reduce en un 30\% el riesgo de desarrollar enfermedad cardiovascular. 


\section{Fundamentación del problema}

Puesto que las enfermedades cardiovasculares constituyen una de las principales causas de morbilidad -mortalidad es lógico que sea de gran interés el análisis de la presencia de los factores de riesgo de padecer este tipo enfermedades, tanto para intentar conocer los posibles mecanismos que afectan al aumento del riesgo, como para poder intervenir precozmente, mediante campañas preventivas, o en su momento con tratamientos terapéuticos.

Los factores de riesgo mayores $e$ independientes para enfermedad cardiovascular son: tabaquismo, hipertensión arterial sistémica, elevación del colesterol total y colesterol de baja densidad (C-LDL), colesterol de alta densidad (C-HDL) bajo, diabetes mellitus, edad avanzada.

La evaluación global de riesgo puede ser clínicamente útil porque nos va a permitir identificar los pacientes de alto riesgo (por ejemplo: aquellos con 2 o más factores de riesgo) que requieran atención e intervención inmediatas. Motivar a los pacientes para mejorar la adherencia en terapias de reducción de riesgo. $Y$ por otra parte, realizar acciones preventivas orientadas a incrementar las conductas de reducción de riesgo en la población.

La naturaleza multifactorial de los fenómenos cardiovasculares hace del proceso de prevención una tarea compleja.

Una vez se haya establecido que el factor está causalmente relacionado con la enfermedad, deben realizarse intervenciones para corregirlo y evaluar el impacto clínico de esa modificación.

E1 tabaquismo, el hipercolesterolemia, y la hipertensión arterial sistémica están causalmente relacionadas con enfermedad coronaria y las intervenciones correspondientes, dejar de fumar, reducir el colesterol, controlar las cifras tensionales, son todas intervenciones benéficas y costo-efectivas tanto en prevención primaria como secundaria:

- Las personas que consumen 20 cigarrillos o más tienen 2 a 3 veces más riesgo de desarrollar enfermedad coronaria, que los que no fuman. Además de aumentar el riesgo de infarto agudo de miocardio, el tabaquismo aumenta la incidencia de muerte súbita, desarrollo de aneurisma aórtico, enfermedad vascular periférica y evento cerebrovascular isquémico. Las personas que dejan de fumar reducen el riesgo de un evento coronario en un $50 \%$ en los primeros 1 a 2 años después de suspender el hábito, y el riesgo se aproxima al de los no fumadores después de 5 a 15 años.

- La hipertensión arterial aumenta el riesgo de infarto agudo de miocardio en $27 \%$ y de evento vascular cerebral en $42 \%$ por cada $7 \mathrm{mmHg}$ de más en la cifra diastólica. La reducción de la cifra diastólica entre 5 y $6 \mathrm{mmHg}$ causa una disminución del $42 \%$ en el riesgo de EVC y del $15 \%$ de eventos coronarios. La magnitud del beneficio y el riesgo de una determinada intervención es esencial en la evaluación de costo-eficacia y en el desarrollo de estrategias preventivas. 


\section{Materiales y métodos}

\section{Población estudiada y diseño del estudio}

Se realizó un estudio de corte transversal, durante los años 2016 y 2017, en una muestra de 59 individuos (48 mujeres y 11 hombres), de rango de edades de 1870 años. La muestra incluye a pobladores de zonas rurales aledañas a las localidades de Corrientes y Chaco donde se instalaron puestos bioquímicos como servicio a la comunidad.

Previo consentimiento informado, se tomaron muestras de sangre venosa, para determinar glucemia y el perfil lipídico (colesterol total, HDL colesterol, LDL colesterol y triglicéridos). En forma paralela se realizó una encuesta, se tomaron medidas antropométricas y de presión arterial.

\section{Encuesta}

Se diseñó un cuestionario (el cual fue previamente validado) en el cual se consideró antecedentes personales, situaciones de la vida cotidiana y estilo de vida (tabaquismo, alcoholismo, sedentarismo, tipo de alimentación, etc.).

\section{Medidas antropométricas}

Se realizaron mediciones de: peso y talla con balanza CAM que posee altímetro telescópico colocado en el centro para que la medida sea precisa. Con los datos obtenidos se calculó el índice de masa corporal (IMC), criterio internacional de obesidad, como la relación existente entre el peso corporal (kilogramos) divido la talla expresada en metros al cuadrado.

\section{Presión arterial}

Se utilizó un tensiómetro digital OMROM modelo HEM-7113, siguiendo las recomendaciones de la Sociedad Argentina de Hipertensión.

\section{Exámenes de laboratorio}

Las muestras de sangre fueron procesadas en el Laboratorio de Bioquímica Aplicada. Se utilizó el autoanalizador IntelligentClinicalChemistryAnalyzer (INCA), obteniendo mediciones de glucemia (Wiener Lab. Glicemia enzimática AA liquida.), colesterol total (Wiener Lab. Colestat enzimático AA liquida.), HDL-colesterol (Wiener Lab. HDL colesterol monofase AA plus.), LDL-colesterol (Wiener Lab. LDL colesterol monofase AA.) y triglicéridos (Wiener Lab. TG color GPO/PAP AA.).Se empleó suero liofilizado (Standatrol S-E 2 niveles - Wiener) como control de calidad.

Se definieron los puntos de corte para algunos FRCV según los siguientes criterios:

IMC: Obesidad $>29,9$

PERFIL LIPIDICO: para el diagnóstico de dislipidemias se consideraron valores

$\mathrm{CT}>200 \mathrm{mg} / \mathrm{dL}, \mathrm{LDLc}>160 \mathrm{mg} / \mathrm{dL}$, TG $>150 \mathrm{mg} / \mathrm{dL}$ y HDLc $<40 \mathrm{mg} / \mathrm{dL}$.

GLUCEMIA: En condiciones de ayuno, se consideró glucemia elevada $>110$ $\mathrm{mg} / \mathrm{dL}$ y posible diabetes no confirmada a valores de glucemia $>126 \mathrm{mg} / \mathrm{dL}$.

PRESION ARTERIAL (PA): PA Sistólica hasta120 mmHg. PA Diastólica hasta $80 \mathrm{mmHg}$. 
Por último se entregaron a los pacientes los resultados de las mediciones antropométricas realizadas con valores de referencia, así como también los valores de laboratorio posteriormente al análisis y valoración de los mismos.

\section{Análisis estadístico}

Todos los datos fueron cargados en planillas de Excel 2010. Los análisis fueron realizados utilizando el software InfoStat 2015 versión estudiantil. Proporciones y desviaciones estándar fueron usados para describir la muestra en el estudio. Se calculó la correlación de Pearson (r) para cada variable antropométrica y variables lipídicas, con un nivel de significancia $\mathrm{p}<0,05$.

\section{Resultados}

De las 59 personas incluidas en el estudio, $81 \%(n=48)$ correspondieron al sexo femenino y $19 \%(\mathrm{n}=11)$ al masculino. La edad promedio fue de 41,5 años.

El análisis estadístico no arrojó diferencias significativas al comparar el comporta- miento entre ambos sexos para la mayoría de los factores de riesgo analizados. Posiblemente esté relacionado al hecho de que la población estudiada no fue homogénea presentando mayoría de mujeres (81\%).

La distribución general de los factores de riesgo cardiovascular en la población de adultos del sector estudiado se presenta en el gráfico No1. De acuerdo a la encuesta realizada se logró recabar que el sedentarismo y la obesidad comparten el primer lugar de prevalencia en la población estudiada.

E1 73\% reportó realizar actividad física menos de 30 minutos, tres veces por semana, lo cual se consideró como indicador de sedentarismo.

E1 46\% de la población analizada presentó un IMC mayor al 29,9\% [IC95\%( 27,83-32,73)] indicando obesidad.

E1 44\% presentó presión arterial sistólica superior a $120 \mathrm{~mm} \mathrm{Hg}$ y/o diastólica superior a $80 \mathrm{~mm} \mathrm{Hg}$. Mientras que el $17 \%$ de los entrevistados declaró ser fumador actual.

E1 20\% de los pacientes refirió padecer de D.M.

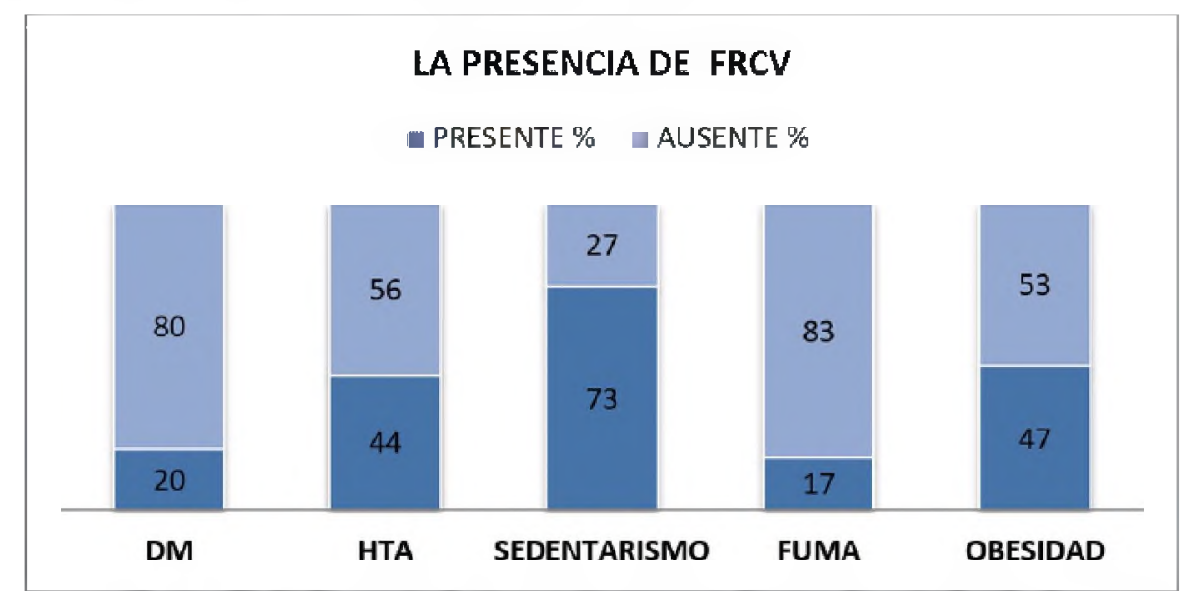

Gráfico $N^{\circ}$ 1: Presencia de factores de riesgo cardiovasculares individuales.

Ref:DM: Diabetes Mellitus-HTA: Hipertensión arterial 
En cuanto al elevado nivel de obesidad blación presentaba sobrepeso, como puede (47\%), se detectó además que $24 \%$ de la po- observarse en el Gráfico No2.

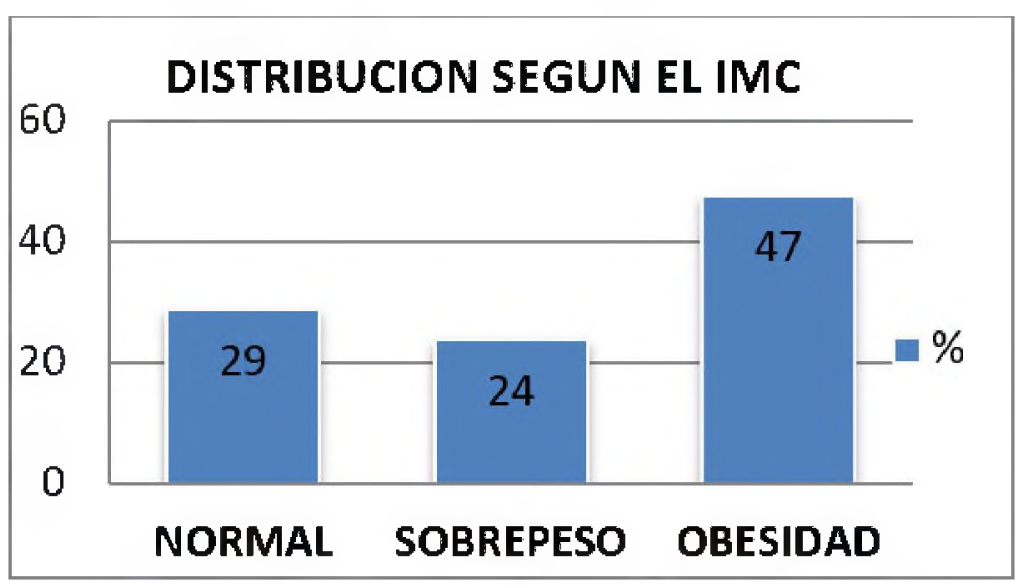

Gráfico No2: Distribución según el IMC

Por otra parte, se estudiaron las variables bioquímicas que pueden producir riesgo a desarrollar enfermedades cardiovasculares, plasmadas en el Gráfico No3.

Las alteraciones que se presentaron en mayor frecuencia fueron la disminución de colesterol en lipoproteínas de alta densidad [cHDL (bigh-densitylipidcholesterol)] $31 \%$ considerando HDLc $<40 \mathrm{mg} / \mathrm{dL}, 110 \mathrm{mg} / \mathrm{d} 1$ [IC95\%(89,94-124,42)].

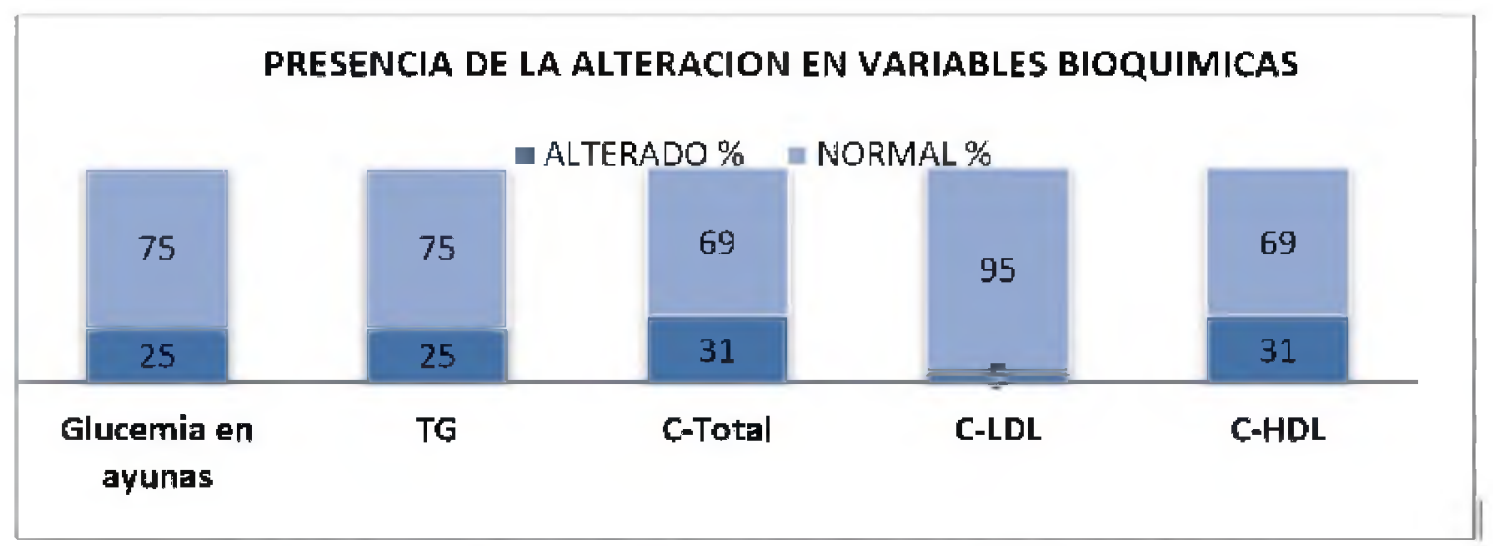

Gráfico No3: Presencia de la alteración en variables bioquímicas individuales.

Ref: TGC: Trigliceridemia-C-total: Colesterol total-C-LDL: colesterol de baja densidad-C-HDL: colesterol de alta densidad 
Al realizarse el análisis de cuantos factores de riesgo presentaba cada individuo estudiado, los FRCV considerados fueron los siguientes: IMC $\geq 29.9$, tabaquismo, sedentarismo, $\mathrm{PAS}>120 \mathrm{mmHg}$ y/o PAD > $80 \mathrm{mmHg}, \mathrm{CT}>200 \mathrm{mg} / \mathrm{dl}$ y glucemia $\geq$ $110 \mathrm{mg} / \mathrm{d} 1$.
E1 5\% de las personas no presentó ninguno de los factores de riesgo analizados, mientras que el 29\% presentó un FRCV, un 27\% presentó dos FRCV y el 39\% presentó tres o más de ellos de forma simultánea.

La asociación más frecuente fue el sedentarismo unido al IMC elevado.

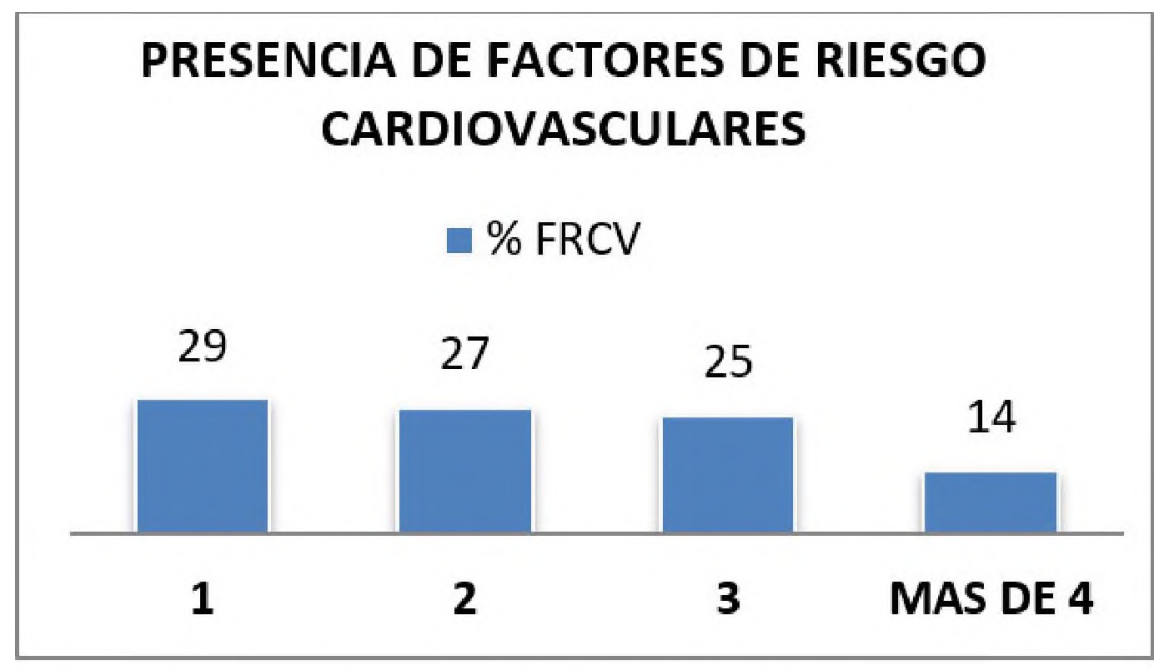

Gráfico No4: Presencia de factores de riesgo acumulados

\section{Discusión}

Este trabajo fue realizado con el objetivo de estudiar y comparar la prevalencia de los diferentes factores de riesgo a desarrollar enfermedades cardiovasculares. De acuerdo con los resultados obtenidos, llama la atención la elevada cifra de obesidad que, incluyendo al sobrepeso, compromete al $71 \%$ de la población. El alto porcentaje de obesidad cobra importancia por ser un factor de riesgo no solo para las enfermedades cardiovasculares, sino también para otras enfermedades crónicas. Aquellos que presentaron sobrepeso, poseen altas probabilidades de desarrollar obesidad en un futuro (OMS, 2000).
E1 73\% reportó realizar actividad física menor de 30 minutos tres veces por semana, lo cual se consideró como indicador de sedentarismo. Los resultados obtenidos concuerdan con lo que dice La Organización Mundial de la Salud al respecto; La OMS afirma que más del 60\% de la población mundial es sedentaria.

La glucemia en ayunas elevada permitió el diagnóstico de diabetes en tres personas, lo que confirma que, en países en desarrollo como Argentina, aún son muchos los diabéticos que desconocen su situación. (Asociación Latinoamericana de Diabetes. Guias ALAD de diagnóstico, control y tratamiento de la diabetes mellitustipo 2. ALAD, 2007). 
En lo que respecta al tabaquismo, el $17 \%$ de la población estudiada refirió ser fumadora, concordando con los resultados obtenidos en Cartagena de Indias ("Búsqueda activa y evaluación de factores de riesgo cardiovascular en adultos jóvenes”), donde la población fue muy similar a la nuestra. Esto puede explicarse por 1a influencia que genera el entorno cultural, lo cual coincide con lo expresado en la Encuesta Nacional de Adicciones 2002.

Analizando presión arterial, vemos que el 44\% presentó presión arterial sistólica superior a $120 \mathrm{~mm} \mathrm{Hg}$ y/o diastólica superior a $80 \mathrm{~mm} \mathrm{Hg}$, concordando con los resultados obtenidos en el trabajo "Síndrome metabólico en personas adultas de la provincia de Corrientes" el cual fue llevado a cabo en la región del NEA al igual que nuestro estudio. Ahora bien, cuando comparamos estos resultados con los obtenidos en trabajos realizados en otros países de la zona, como por ejemplo el estudio realizado en Cartagena de Indias ("Búsqueda activa y evaluación de factores de riesgo cardiovascular en adultos jóvenes") no sucede lo mismo, ya que en esa población se vio que el 9\% presentó HTA, podemos decir entonces que este FRCV en Argentina se encuentra muy por encima de la media de la región.

Para la realización de este estudio se incluyeron los FRCV tradicionales dado que son datos simples de obtener(a través de la realización de una encuesta), medición de la presión arterial y determinaciones bioquímicas a las cuales tienen acceso los laboratorios de pequeña y mediana complejidad.
Por otra parte, no podemos dejar de mencionar que en el último tiempo se observó que muchos ataques cardíacos ocurren a personas con bajo riesgo de enfermedad cardiovascular; casi el $80 \%$ de los pacientes coronarios tienen niveles de colesterol similares a los de la población aparentemente sana. Buscando una explicación a este fenómeno se han hallado otros factores asociados a la enfermedad vascular y se los ha denominado nuevos factores de riesgo cardiovascular ("Nuevos Factores de Riesgo Cardiovascular" Alberto Lorenzatti, Luis A. Guzman, Carlos A. Cuneo)

Dichos factores fueron identificados a partir de la investigación etiopatogénica de la aterosclerosis y respaldados en observaciones clínicas, epidemiológicas y de laboratorio. Varios de estos factores son considerados, en la actualidad, predictores de riesgo independiente.

Homocisteína, fibrinógeno, subclases de LDL (LDL pequeñas y densas), Lipoproteína(a) [Lp(a)], proteína C reactiva (PCR), inhibidor del activador tisular del plasminógeno 1 (PAI-1), factor tisular, viscosidad plasmática aumentada, anticuerpos antifosfolipídicos, infecciones por Chlamydia pneumoniae, Helicobacter pylori, herpes simple y citomegalovirus, etc., por mencionar sólo algunos de los más estudiados, son parte de la cada vez más amplia lista de factores de riesgo emergentes.

Lamentablemente realizar estudios epidemiológicos de gran escala teniendo en cuenta estos factores de riesgo emergentes en este momento sería casi imposible dado su alto costo. 


\section{Conclusión}

La alta prevalencia de factores de riesgo cardiovascular en adultos jóvenes remarca la importancia de realizar campañas de concientización y prevención encaminadas a reducirlos.

Un alto porcentaje de la población incluida en el estudio presentó obesidad y sedentarismo esto señala la necesidad de educar a la población para mejorar los hábitos alimenticios y aumentar la actividad física.

Estos resultados se convierten en una línea de base para el estudio de factores de riesgo cardiovascular y enfermedades cardiovasculares, que puede ser utilizada para derivar macro-proyectos que beneficien a la población.

\section{Bibliografia}

- Alicia Norma Alayón, Saudith Ariza, Karen Baena, Lina Lambis, Lina Martínez, Lourdes Benítez (2007). Búsqueda activa y evaluación de factores de riesgo cardiovascular en adultos jóvenes, Cartagena de Indias. Biomédica Revista del Instituto Nacional de salud Colombia.

- Asociación Latinoamericana de Diabetes. Guías ALAD de diagnóstico, control y tratamiento de la diabetes mellitus tipo 2. ALAD, 2007.
- UffeRavnskov, David M Diamond, Rokura Hama, TomohitoHamazaki, Björn Hammarskjöld, Niamh Hynes, Malcolm Kendrick, Peter H Langsjoen, AseemMalhotra,LucaMascitelli, Kilmer S McCully, Yoichi Ogushi, Harumi Okuyama, Paul J Rosch, Tore Schersten, Sherif Sultan, Ralf Sundberg (2016). Lack of an association or an inverse association between low-densitylipoprotein cholesterol and mortality in the elderly: a sys- 
tematic review.Published by group.bmj.com

- Dra. Yecdany Licea López; Dr. Ismael Ferrer Herrera; Dr. René A. Báez Medina; Dr. Alberto A. Llanes Rodríguez; Dr. Ismael Ferrer Tan(2006).Estratificación del riesgo cardiovascular en hipertensos atendidos por médicos de la familia. Centro Provincial de Ciencias Médicas de Camagüey Camagüey, Cuba.

- Consenso Nacional para el Diagnóstico y Manejo de la Hipertensión Arterial Sistémica. Clínicas Colombianas de Cardiología 1998; 1: 115-175.

- Executive Summary of the Third Report of the National Cholesterol Education Program (NCEP) Expert panel on Detection, Evaluation and Treatment of High Blood Cholesterol in Adults (Adult Treatment Panel III). JAMA 2001; 285: 2486-2497.

- Alberto Lorenzatti, Luis A. Guzman, Carlos A. Cuneo (1999). Nuevos Factores de Riesgo Cardiovascular. RevFedArgCardiol 28: 539-544

- Bustos M Patricia, Amigo C Hugo, Arteaga Ll Antonio, Acosta B Ana María, Rona Roberto J. (2003). Factores de riesgo de enfermedad cardiovascular en adultos jóvenes. Revista médica de Chile, 131(9), 973-980.

- Guías de la sociedad argentina de hipertensión para el diagnóstico, estudio, tratamiento y seguimiento de la hipertensión arterial. Ministerio de salud presidencia de la nación. (2016). Programa nacional de control contra el tabaco.

- Amit Sachdeva, MD, a Christopher P. Cannon, MD,b Prakash C. Deedwania, MD,c
Kenneth A. LaBresh, MD,d Sidney C. Smith, Jr, MD,e David Dai, MS,f Adrian Hernandez, MD,f and Gregg C. Fonarow,. (2009)Lipid levels in patients hospitalized with coronaryartery disease: An analysis of 136,905 hospitalizationsin Get With The Guidelines. MD a on behalf of the GWTG Steering Committee and Hospitals Los Angeles and San Francisco, CA; Boston and Waltham, MA; andChapel Hill and Durham, NC

- Morlans Paz Jesus A. La obesidad: factor de riesgo de la cardiopatía isquémica. Instituto de cardiología y cirugía cardiovascular.

- Organización panamericana de la salud, oficina regional de la organización mundial de la salud. Hipertensión arterial.

- Pineda Barria Sandra Denise. (2009). Estudio de los Factores de Riesgo Cardiovascular en Estudiantes de Primer Año de las Carreras de Pregrado del Campus Isla Teja de la Universidad Austral de Chile, Año 2007 (tesis de grado). Universidad Austral, facultad de ciencias escuela de Quimica y Farmacia, Valdivia Chile.

- Esp. Goicoechea, Patricia N.;Dra. Aguirre, María Victoria; Bqca. Serrano, Natalia A.; Bqca. Martinez, Silvina M.;Bqco. Leyes Pedrozo, Ariel E.; Esp. Ghione, Lucrecia E. Esp. Gauna Pereira, María del Carmen; Esp. Reyes, Alberto Daniel. (2013). Síndrome metabólico en personas adultas de la provincia de Corrientes: análisis según género, procedencia y grupo etario. Extensionismo, innovación y transferencia tecnológica. Claves para el desarrollo. 